\title{
A Rare Case of Ileo-ileal Fistula within a Strangulated Incisional Hernia "Fistulation by the Sneaky Plastic Perforator"
}

Dewaraj Velayudhan, Sarmukh Singh \& Azmi Hassan

Department of General Surgery, Hospital Sultan Haji Ahmad Shah, Temerloh, Pahang, Malaysia.

Perforation of a hollow viscus by a foreign body is seldom observed in clinical practice. Ileo-ileal fistula secondary to perforation by ingested plastic material is a rare entity. Accidental ingestion of a foreign body is often encountered in clinical practice. However, intestinal perforation due to such a cause is rare because the swallowed foreign body usually advances through the gastrointestinal tract without any problems and is excreted via faeces. Only $1 \%$ of ingested object results in gastrointestinal system perforation [1]. Commonly the materials would be plastic, glass or iron with sharp edges. However, in this case it was a plastic sheath. Herein, we present a patient who is prisoner and drug abuser admitted for suspected strangulated incisional hernia, in view of history of previous surgery done. However, on-table a plastic sheath was discovered to be causing an ileo-ileal fistulation with perforation. This case stands out due to the rarity of its entity, peculiarity of its clinical presentation and complexity in managing it. This case highlights that suspicion of foreign body ingestion is imperative in diagnosing and managing acute abdominal pain cases among prisoners and drug abusers.

Keywords: Ileo-ileal fistula, Perforation, Foreign body, Prisoner.

\section{Case Study}

\section{Case Presentation}

A 40 years old Malay gentleman, who is a prisoner with the history of being an ice-chaser presented with non-radiating, colicky abdominal pain over the right hypochondrium and umbilical regions for the past four months. There was aggravation of pain for the past two days. Pain was associated with non-projectile vomiting with food content. Patient denied fever and altered bowel and bladder habits. Upon further history-taking, it is learnt that patient has undergone an operation in March 2017 for presumptive diagnosis of acute appendicitis and was suspected to have a caecal tumour, for which a right-hemicolectomy with primary anastomosis was performed. However, histopathology report later showed benign ulcer at ceacum. Patient then defaulted his subsequent follow-up.

On physical examination, he was dehydrated. Upon per abdomen examination, a well-healed Lanz incision and midline-laparotomy scars were noted. Abdomen was tender and fullness over the right iliac fossa (previous Lanz incision) with erythematous skin changes was appreciated. Bowel sounds were present on auscultation. Digital rectal examination was unremarkable. Laboratory investigation revealed leukocytosis and microcytic hypochromic anemia. We proceeded with ultrasound abdomen and noted herniated bowel and omental fat with suspicious bowel strangulation in the area of previous Lanz incision scar. Our provisional diagnosis was strangulated incisional hernia.

We proceeded with exploratory laparatomy with double barrel stoma creation. Intraoperatively, there was an incisional hernia defect of $2 \mathrm{~cm}$ at the previous Lanz incision site. The sac contained small bowels that formed a knuckle with fistulation between them. Within the fistula, there was a small, thin and elastic white plastic material. There was also perforation with localized pus. 
Post operatively, patient was nursed in Intensive Care Unit. In view of the severe sepsis secondary to perforated ileo-ileal fistula, patient eventually succumbed.

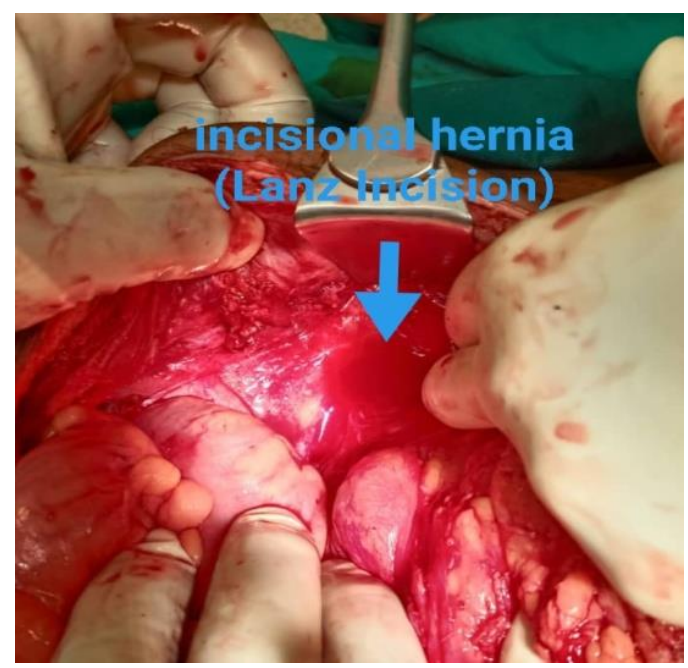

Fig.1 Intraoperative findings shows incisional hernia with a defect size of $2 \mathrm{~cm}$. Hernia sac contained small bowels forming a knuckle

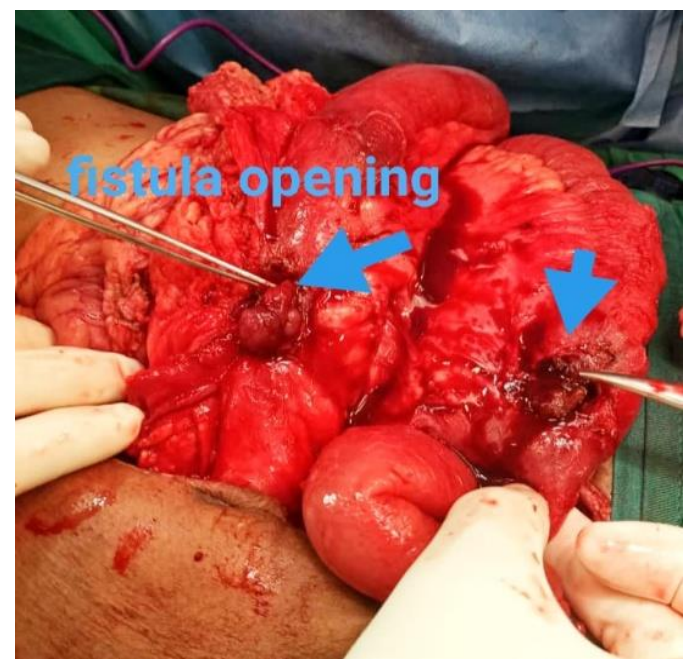

Fig.2 Picture showing the fistulation between small bowels of the knuckle within the hernia sac, with perforation containing localized pus

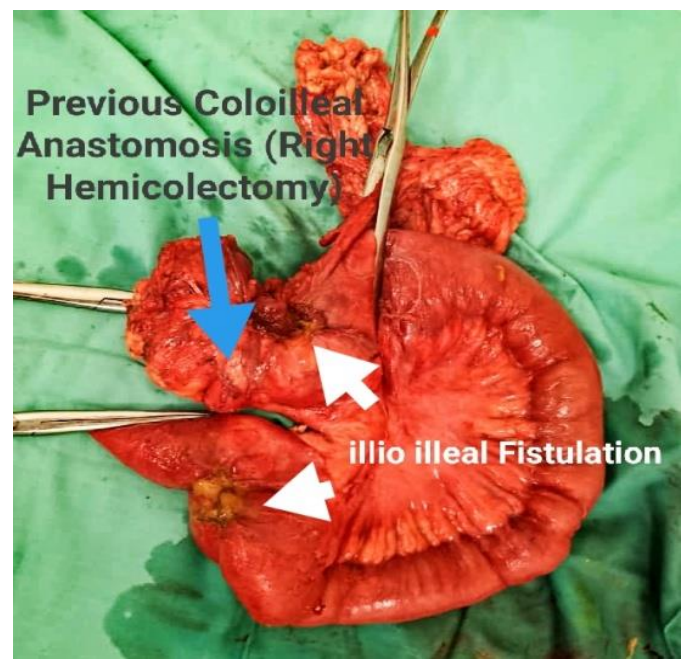

Fig.3 Picture showing resected small bowel (100 cm from DJ flexure) 


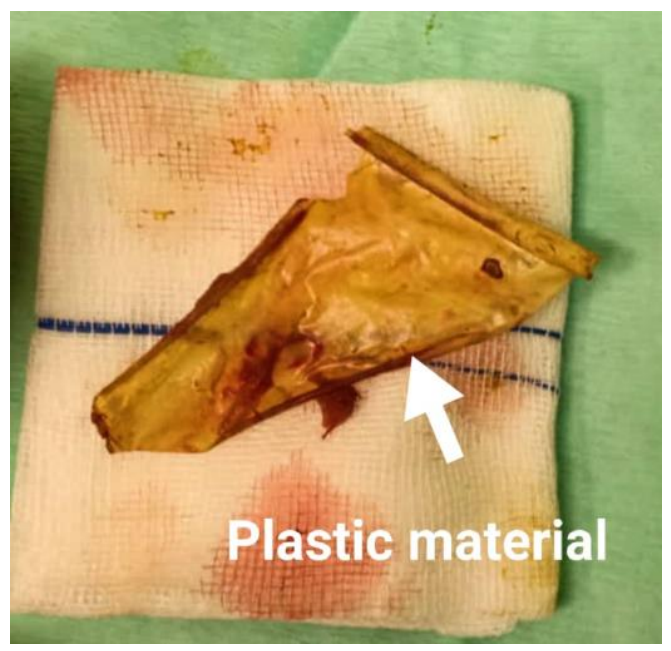

Fig.4 Picture of a small, thin and elastic white plastic material found within the fistulation

\section{Discussion}

The word fistula is derived from Latin, which means 'pipe' [2]. A fistula is an abnormal connection between two epithelized surfaces. Internal intestinal fistulas are relatively harmless. Symptoms only manifest if there is significant obstruction or sepsis. Similar to our patient who presented with symptoms of obstruction. The aggravation of his sepsis, despite localised minimal pus is explained by his poor nutritional and immunocompromised status as he was a chronic drug abuser.

The etiology of most gastro-intestinal fistulas occurs as a complication from surgeries. Some fistulas can also be attributed to inflammation (Crohn's disease) and infective causes (intestinal tuberculosis). However, in our case the fistula is likely to be attributed to a foreign body, which is a rare etiology. It is even more rare in adults as incidences of foreign body ingestion are more common in pediatric age group [3]. Hence, post-operation, further history was taken from the patient. It was deduced that patient used to be a smuggler and had swallowed condoms filled with drugs in it. The plastic material found could be a retained piece of condom. Though plastic is an inert chemical, prolonged trapping within the hernial sac could have incited chronic inflammatory process and caused perforation. The perforation in turn further aggravated the inflammation and encouraged the migration of granulation tissue, fibrin and adjacent bowel loops [4]. Over a period of time, a fistula had formed within the adjacent bowel loops. This hypothesis is supported by the finding of the localized pus collection within the fistula.

Based on our literature review, only $1 \%$ of foreign body ingested caused gastrointestinal perforation [5]. Most pass-through gastrointestinal system without any problems and are excreted via faeces. Among those that cause perforation are usually long and sharp-edged objects. However, in our case the foreign body was a soft plastic material (likely condom). The common sites of perforation are usually the terminal ileum and recto-sigmoid regions due to its anatomic angulation [4]. However, in our case perforation occurred in ileum which is an uncommon location. Since the loops of bowel within the knuckle had a sharp angulation too, this explains the perforation.

Ingestion of foreign body is a common encounter in clinical practice [5]. Most often it is accidental ingestion, that too are more common among paediatric age group. Approximately $80 \%$ of foreign body ingestion cases in 
paediatric are of accidental in nature [6]. Whereas in adults, accidental ingestion is usually seen in elderly age group and patients with learning disabilities and alcohol dependence [7]. Intentional ingestions are usually seen among adult patients with underlying psychiatric disorders, suicidal intention and prisoners. The history to be elicited is challenging especially in intentional ingestions as patients tend to be reluctant to share the truth. It has been trickier here as patient's ingestion has been an act against the law. Even in the retrospective history-taking during the post-operative period, patient was in denial. However, after further assurance patient confessed that he has been a drug mule by ingesting condom-filled drugs before but was afraid in view of fear towards the consequences of law. The key point is to have a differential of foreign body ingestion as an etiology when handling any case of abdominal pain among imprisoned patients [8]. The motives behind ingestions vary, hence detailed history taking with good patient rapport are vital [9].

The dilemma in diagnosing foreign body ingestion is real. A generalized protocol is necessary however, the flexibility of customization should be given on case-to-case basis. Abdominal x-ray, computed tomography (CT) and ultrasonography (US) are useful tools in evaluation of abdominal pain. The pros and cons of each utility should be thoroughly analyzed. Abdominal x-ray is useful to detect air under diaphragm (as a result of perforation) or dilated bowels loops (due to obstruction). However, only $15.9 \%$ of foreign object perforation cases present with free air under diaphragm following perforation due consequent covering of the perforation by an inflammatory mass [4]. At such, CT scan is another option that is highly sensitive especially in retrospective evaluation. A study done showed CT being sensitive at $7.4 \%$ in picking up a fish bone and sensitivity can be increased to $100 \%$ upon retrospective evaluation [10]. However, in our case the retrospective interpretation of CT did not reveal the plastic foreign body as it is radiolucent. This highlights the importance of US in detecting foreign bodies that are not radio-opaque. US also has the upper hand of being highly flexible with high reproducibility, low in costing and with complete avoidance of radiation exposure [11]. US in this instance, did not reveal the foreign body but instead showed target sign with possible intussusception. This misleading point is attributed to the altered anatomy of bowels, adhesions and incisional hernia secondary to prior surgery. Laparoscopy is another vital option as part of both diagnostic and therapeutic tool [12]. We opted for exploratory laparotomy instead of laparoscopy as the provisional diagnosis was intestinal obstruction secondary to strangulated incisional hernia.

\section{Conclusion}

Foreign body ingestion as an aetiology should always be ruled out in acute abdomen cases among adults of high-risk groups such as prisoners and drug abusers. A good rapport and tactful approach are essential to gain patient's confidence. The combination of a good history-gathering skill and rational suspicion of a clinician is the most supreme diagnostic tool.

\section{References}

1. Pinero M.A, Fernández H.J.A, Carrasco P.M, Riquelme J, Parrila P.P., Intestinal perforation by foreign bodies. Eur J Surg 2000; 166: 307- 309.

2. David E Stein, Rebecca B Hasley, Aisya S Ahmad, Intestinal Fistulas, Medscape https://emedicine.medscape.com/article/179444-overview 
3. Andrew J.R, Jane B, Ronald L.E.T, Magnet induced Perforated Appendicitis and ileo-caecal fistula formation, Ulster Med J. 2009 Jan 78 (1) 4-6

4. Goh B.K, Chow P.K, Quah H.M, Ong H.S, Eu K.W, Ooi L.L., Perforation of the gastrointestinal tract secondary to ingestion of foreign bodies. World J Surg 2006; 30; 372-377.

5. May B, Mark E.O, Niall G., Foreign body ingestion in prisoners - The Belfast Experience, Ulster Med J. 2008 May; 77(2): 110-114

6. Ruslan Abdullayev, Mahmut Aslan, Ileal Perforation by an odd foreign object, Ulus Cerrahi Derg 2015; 31: 107-9

7. Pavlidis T.E, Marakis G.N, Triantafyllou A, Psarras K, Kontoulis T.M, Sakantamis A.K., Management of ingested foreign bodies. How justifiable is a waiting policy?, Surg Laparosc Endosc Percutan Tech, 2008 Jun; 18(3): 286-7

8. Eisen G.M, Baron T.H, Dominitz J.A, Faigel DO, Goldstein J.L., American Society for Gastrointestinal Endoscopy, Guideline for the management of ingested foreign bodies, Gastrointest Endosc. 2002 Jun; 55(7):802-6 9. David C.E, Thomas R.W, Christian D.J, Andrew J.O, Stanislaw P.S., Intentional Ingestions of foreign objects among prisoners: A review, World J. Gastrointest Endosc. 2015 Mar 16; 7(3): 162-168

10. Goh B.K, Tan Y.M, Lin S.E, Chow P.K, Cheah F.K, Ooi L.L, et al. CT in the pre-operative diagnosis of fish bone perforation of the gastrointestinal tract, JR Am J Roentgenol 2006; 197: 710-714

11. Drakonaki E, Chatzioannou M, Spiridakis K, Panagiotakis G. Acute abdomen caused by a small bowel perforation due to a clinically unsuspected fish bone. Diagn IntervRadiol 2011; 17:160-162

12. Law W.L, Lo C.Y. Fishbone perforation of the small bowel: laparoscopy diagnosis and laparoscopically assisted management. Surg Laparasc Endosc Percutan Tech 2003; 13: 392-393 